\title{
Nuclear-magnetic-resonance imaging of leaves of Mesembryanthemum crystallinum L. plants grown at high salinity
}

\author{
L. Walter ${ }^{1}$, A. Balling ${ }^{1}$, U. Zimmermann ${ }^{1}$, A. Haase ${ }^{2}$, and W. Kuhn ${ }^{3}$ \\ ${ }^{1}$ Lehrstuhl für Biotechnologie der Julius-Maximilians-Universität Würzburg, Röntgenring 11, D-8700 Würzburg, \\ ${ }^{2}$ Max-Planck-Institut für Biophysikalische Chemie, Am Fassberg, D-3400 Göttingen, and \\ ${ }^{3}$ Bruker Analytische Messtechnik GmbH, Am Silberstreifen, D-7512 Rheinstetten, Federal Republic of Germany
}

\begin{abstract}
Differences in water binding were measured in the leaf cells of Mesembryanthemum crystallinum L. plants grown under high-salinity conditions by using nuclear-magnetic-resonance (NMR) imaging. The 7-Tesla proton NMR imaging system yielded a spatial resolution of $20 \cdot 20 \cdot 100 \mu \mathrm{m}^{3}$. Images recorded with different spin-echo times (4.4 $\mathrm{ms}$ to $18 \mathrm{~ms}$ ) showed that the water concentrations in the bladder cells (located on the upper and lower leaf surface), in the mesophyll cells and in the water-conducting vessels were nearly identical. All of the water in the bladder cells and in the waterconducting vessels was found to be mobile, whilst part of the water in the mesophyll cells was bound. Patches of mesophyll cells could be identified which bound water more strongly than the surrounding mesophyll cells. Optical investigations of leaf cross-sections revealed two types of mesophyll cells of different sizes and chloroplast contents. It is therefore likely that in the small-sized mesophyll cells water is strongly bound. A long-term asymmetric water exchange between the mesophyll cells and the bladder cells during Crassulacean acid metabolism has been described in the literature. The high density of these mesophyll cells in the lower epidermis is a possible cause of this asymmetry.
\end{abstract}

Key words: Crassulacean acid metabolism - Leaf (water binding) - Mesembryanthemum - Mesophyll (water binding) - Nuclear magnetic resonance microscopy - Water, free and bound

\section{Introduction}

In the last decade, spatially localised proton nuclear-magnetic-resonance (NMR) imaging has be-

Abbreviations: $\mathrm{CAM}=$ Crassulacean acid metabolism $; \mathrm{NMR}=$ nuclear magnetic resonance; $\mathrm{T}_{\mathrm{E}}=$ spin-echo time come an established method in medical diagnosis. The ${ }^{1} \mathrm{H}-\mathrm{NMR}$ imaging is a non-invasive method and allows in-vivo observations of changes in water content and binding in an organ or tissue. For whole-body imaging, magnets of an inner diameter of $1 \mathrm{~m}$ are used. Economic and practical constraints limit the field strengths to a maximum of 4 Tesla (170 MHz resonance). With these magnets the spatial resolution is only of the order of $1 \mathrm{~mm}^{3}$. However, the resolution has recently been extended to volume elements of less than $0.012 \mathrm{~mm}^{3}$ by using a modified whole-body NMR-imaging system (Johnson et al. 1987). Magnetic resonance microscopy on a tissue or even at a cellular level is, therefore, conceivable (Aguayo et al. 1986; Bottomley et al. 1986).

Several authors have recently reported on NMR sub-millimetre-imaging of plant tissues (Eccles and Callaghan 1986; Connelly et al. 1987; Johnson et al. 1987). However, the field strengths and gradients used in these studies were not high enough to achieve an in-plane resolution that approaches the dimensions of a single cell in a plant tissue.

In this communication we report on high-resolution NMR microscopy of plant tissue cells by means of a 7-Tesla, 89-mm-bore spectrometer. The investigations were performed on leaves of Mesembryanthemum crystallinum L. plants in which Crassulacean acid metabolism (CAM) had been induced by hydroculture of the plants in the presence of high salinity (Lüttge et al. 1978; Winter 1985). The lower and upper epidermes of the leaf of this plant are covered by bladder cells which have a volume between $0.05 \mathrm{~mm}^{3}$ and $0.8 \mathrm{~mm}^{3}$. From pressure-probe measurements it is known that these large bladder cells function as water-exchange reservoirs during the malate accumulation and degradation which occurs in the much smaller mesophyll cells (average volume $0.7 \cdot 10^{-3} \mathrm{~mm}^{3}$; 
Steudle et al. 1975; Rygol et al. 1986, 1989; Zimmermann 1988). Moreover, there is evidence that the water exchange predominantly occurs between the mesophyll cells and the bladder cells of the upper epidermis, but not between those of the lower epidermis.

The signal-to-noise ratio of the NMR-imaging spectrometer was sufficiently high to distinguish small differences in proton signal intensities between volume elements of $4 \cdot 10^{-5} \mathrm{~mm}^{3}$. Therefore, the resolution was high enough to study spatial differences in water content and binding and to correlate the NMR imaging with the morphological structure of this tissue.

\section{Theory}

In the conventional NMR experiment a magnetic resonance signal is obtained by exposing the tissue to a strong homogeneous magnetic field and a radio-frequency field. The magnetic field induces the protons to precess synchronously about the field vector at the so-called Larmor frequency. Application of a $90^{\circ}$ radio-frequency pulse at the Larmor frequency shifts the precessing protons away from the equilibrium position. In this process the protons absorb energy which is later reemitted. The reemitted signal can be spatially localized to create a digital image by application of a sequence of magnetic gradients (Lauterbur 1973).

The magnitude of transverse magnetization created by the NMR-imaging sequence determines the NMR signal intensity of each image element. The time-dependent amplitude of the measured NMR signal can be calculated from the Bloch equations (Bloch 1946). Two characteristic relaxation times describe the magnetization created by a radio-frequency pulse. The spinspin relaxation time $\left(\mathrm{T}_{2}\right)$ describes the time-dependent decay of the NMR signal, the spin-lattice relaxation time $\left(T_{1}\right)$ describes the recovery of the original equilibrium of the spin sys-
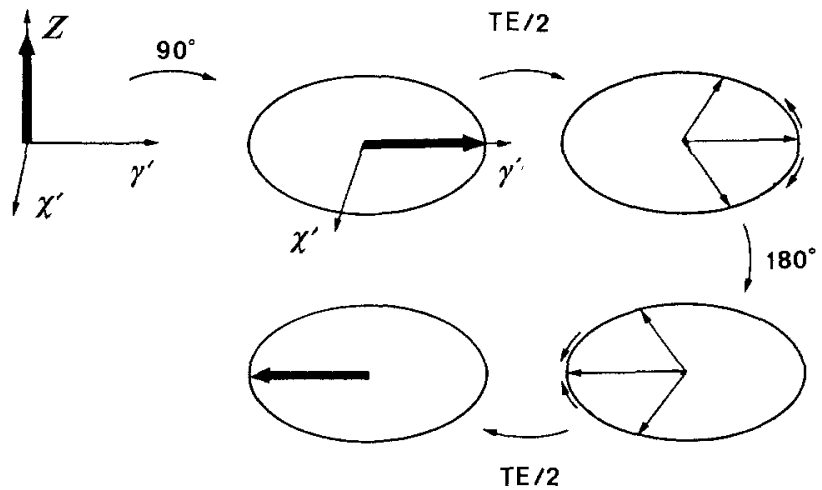

Fig. 1. Schematic vector diagram showing the NMR spin-echo experiment. The vectors represent the nuclear moments at different times within the experiment. A $90^{\circ}$ pulse tilts the nuclear moments so that they lie along the $\mathrm{y}^{\prime}$-axis. During the time interval $\mathrm{T}_{\mathrm{E}} / 2$ the nuclear moments dephase due to inhomogeneities of the magnetic field within the sample. The $180^{\circ}$ pulse inverts the direction of the nuclear moments within the $\mathrm{x}^{\prime}, \mathrm{y}^{\prime}$ plane while they continue to precess. After the second time interval $T_{E} / 2$ they refocus on the $y^{\prime}$-axis resulting in the NMR spin-echo signal tem. The NMR spin echo is obtained by the application of two radio-frequency pulses separated by a time interval of $T_{E} / 2$, where $T_{E}$ is the spin-echo time, with a first $\pi / 2$ pulse and a second $\pi$ pulse. The spin-echo sequence can be expressed as:

$\pi / 2-\mathrm{T}_{\mathrm{E}} / 2-\pi-\mathrm{T}_{\mathrm{E}} / 2-$ spin-echo.

The formation of the NMR spin-echo signal is illustrated in Fig. 1. The initial $90^{\circ}$ pulse tilts the magnetization away from its equilibrium position parallel to the magnetic field axis into the $\mathrm{x}, \mathrm{y}$-plane perpendicular to the magnetic field. The nuclear magnetic moments of the same species, e.g. ${ }^{1} \mathrm{H}$ nuclei of water molecules will remain static along one axis in a reference frame rotating with the NMR resonance frequency. However, as a result of magnetic-field inhomogenities, nuclei of different regions within the sample have slightly different frequencies. Therefore, the magnetic moments of the nuclei will dephase and the resulting measureable NMR signal will decrease. After a time interval $T_{E} / 2$ a $180^{\circ}$ pulse is applied which inverts the various components of the magnetic moments. As a result of this pulse the magnetization will refocus after a second time interval $T_{E} / 2$, giving the spin-echo NMR signal. The refocussing of the magnetic moments will be incomplete if there are irreversible relaxation processes. These can be the diffusion of molecules within inhomogeneous magnetic fields or intramolecular dipolar interactions between different nuclear moments.

Assuming exponential $T_{1}$ and $T_{2}$ relaxation, the intensity (I) of the spin-echo signal can be calculated from the Bloch equations:

$\mathrm{I} \approx \mathrm{N}_{\mathrm{H}}\left(1-\exp \left(-\mathrm{T}_{\mathrm{R}} / \mathrm{T}_{1}\right)\right) \exp \left(-\mathrm{T}_{\mathrm{E}} / \mathrm{T}_{2}\right)$

where $N_{H}$ is the proton density, and $T_{R}$ is the repetition time of the experiment, i.e. the time interval between the $\pi / 2$ pulse and the first pulse of the following experiment. Therefore, the signal intensity of a spin-echo imaging experiment is related to tissue-specific parameters, e.g. the proton density $\mathrm{N}_{\mathrm{H}}$ and the relaxation times $T_{1}$ and $T_{2}$.

The influence of these parameters on the signal intensity can be observed by the acquisition of a series of different spinecho images each obtained using different experimental values of $T_{R}$ or $T_{E}$. For example, if the repetition time $T_{R}$ is long compared with the longest $T_{1}$ relaxation time, the signal intensity becomes dependent on $T_{2}$ relaxation, and by using short values of $T_{E}$ the signal intensity is dependent on the proton density only.

The proton NMR signal of biological tissues contains information about tissue water distribution and water mobility. The $T_{2}$ relaxation time of the water signal describes the loss of transverse magnetization by spin-spin interaction. $\mathrm{T}_{2}$ relaxation is more efficient in large molecules or in water molecules bound to macromolecules giving rise to short values of $T_{2}$ in the range of a few microseconds up to a few milliseconds, whereas the $T_{2}$ values of free water molecules are of the order of a few seconds.

Differences in $\mathrm{T}_{2}$ values of biological tissues are interpreted in terms of the differences of the ratio of "free water" to "bound water". This ratio is dependent on the protein concentration. A strong linear dependence of the concentration of proteins to the relaxation rate $1 / \mathrm{T}_{2}$ can be seen in most cases (Schuhmacher et al. 1987). It is generally accepted that tissue water exists in at least two compartments in a rapid exchange relative to the NMR observation time. The first and major compartment comprises unbound, free water molecules and the second compartment comprises water molecules hydrogenbonded to large molecules (e.g. proteins) or hydration layers (Bottomley et al. 1984). Therefore, regions with long values of $\mathrm{T}_{2}$ have a high concentration of free water molecules, while 
regions with short $T_{2}$ values have a high concentration of bound water molecules (Fullerton et al. 1982).

Equation 2 shows that long values of $T_{R}$ and extremely short values of $\mathrm{T}_{\mathrm{E}}$ have to be used to measure the regional distribution of water in biological tissues $\left(\mathrm{N}_{\mathrm{H}}\right)$ and that somewhat larger ratios of $T_{E} / T_{2}$ give access to the information on regions containing free water and bound water molecules.

\section{Material and methods}

Plant material. Plants of Mesembryanthemum crystallinum $\mathbf{L}$ were grown in climatic chambers from seeds in potting soil under a $12 \mathrm{~h}$ light- $12 \mathrm{~h}$ dark regime. The photon fluence rate was $400 \mu \mathrm{mol} \cdot \mathrm{m}^{-2} \cdot \mathrm{s}^{-1}$. The relative humidity was adjusted to $50 \%$ and $80 \%$ during the light and dark periods, respectively. Daytime temperatures were about $24^{\circ} \mathrm{C}$, nighttime temperatures about $18^{\circ} \mathrm{C}$

For all experiments, plants were transplanted in aerated hydroculture solutions after four weeks and were further grown under the same conditions. For hydroculture, modified Johnson's solution was used (Winter 1973) and every $5 \mathrm{~d}$ the medium was replaced by fresh solution.

For measurements under CAM-conditions, CAM was induced in the plants, when the area of the third foliar leaf reached about $1 \mathrm{~cm}^{2}$, by exposing the plants stepwise to increasing concentrations of $\mathrm{NaCl}$ up to a final concentration of $400 \mathrm{mM}$.

Nuclear-magnetic-resonance microscopy. A Bruker AM-300 widebore Spectrometer equipped with a 7-Tesla magnet of $8.9 \mathrm{~cm}$ bore was used. The spectrometer includes a commercial Bruker micro-imaging accessory. A 10-mm saddle-shaped NMR coil was tuned to $300 \mathrm{MHz}$ for excitation and reception of ${ }^{1} \mathrm{H}-\mathrm{NMR}$ signals. The NMR probe contained coils for the generation of constant magnetic field gradients. Gradient strengths of up to $70 \cdot 10^{-4}$ Tesla $\cdot \mathrm{cm}^{-1}$ could be switched within less than 100 us.

A multi-slice single-echo two-dimensional Fourier-transform-imaging sequence was used. The spin-echo time $\left(\mathrm{T}_{\mathrm{E}}\right)$ was varied between $4.4 \mathrm{~ms}$ and $18 \mathrm{~ms}$, the repetition time $\left(T_{R}\right)$ was adjusted to $1 \mathrm{~s}$. Four averages were needed to improve the image quality, resulting in a measuring time of about $20 \mathrm{~min}$ A $6.6 \mathrm{~mm}^{2}$ field-of-view was observed using a 256.256 matrix of data points. The in-plane spatial resolution is proportional to the ratio of the digital resolution $(1 / 256 \mathrm{~Hz})$ to the gradient strengths. However, the slice thickness of the imaging plane is proportional to the ratio of the frequency bandwidth of the radio-frequency pulse to the gradient strengths. Using the appropriate equations (Mansfield and Morris 1982) and values for these parameters, the in-plane spatial resolution is calculated to be $20.20 \mu \mathrm{m}^{2}$ and the slice thickness to be $100 \mu \mathrm{m}$.

For measurements, a small leaf of the fifth foliar leaf pair of 10-week-old $M$. crystallinum, grown in hydroculture, was placed in an NMR tube of $10 \mathrm{~mm}$ inner diameter. It was not necessary to clamp the leaf. The tube was mounted in the center of the coil. No further preparation was needed.

Intact plants could not be used because of the small dimensions of the NMR tube. Excision of the leaf had no significant effects on the water status of the tissue since NMR measurements over several hours did not show any change in the contrast of the images. In addition, turgor-pressure measurements in individual cells of the leaf (by means of the pressure probe) revealed constant turgor pressure (data not shown; see also Rygol et al. 1986, 1989; Zimmermann et al. 1980). Evaporation effects during the experiments could therefore be excluded
Thick sections. Leaves of the fourth foliar leaf pair of plants grown in hydroculture were used. Sections perpendicular to the main vein of the leaf were cut with a razor blade. Sections parallel to the surface of the leaf were also made.

Semi-thin sections. Pieces of leaves of plants $\left(2 \cdot 4 \mathrm{~mm}^{2}\right)$ grown in salt-added hydroculture were fixed in glutaraldehyde solution $(6.25 \%, \mathrm{pH} 7.2)$ in titrisol (TM; Merck, Darmstadt, FRG) for $2 \mathrm{~h}$. After washing in the same buffer, samples were postfixed in $2 \% \mathrm{OsO}_{4}$ (barbital-buffered, $\mathrm{pH} \mathrm{7.2)}$ for $2 \mathrm{~h}$, washed in buffer and dehydrated in a graded ethanol serie. After intermediate rinsing in propylene oxide, samples were imbedded in Epon 812 (TM; Serva, Heidelberg, FRG). Semithin sections (1.5 $\mu \mathrm{m}$ thickness) were made with a glass knife on a Reichert ultramicrotome, stained with Mallory's azure/methylene blue and observed by microscope.

\section{Results}

The proton NMR spectrum contained one resonance line corresponding to the NMR signal of free water within the object. Figure 2 shows a representative proton spin-echo NMR image of a leaf of $M$. crystallinum using a spin-echo time of $18 \mathrm{~ms}$. The figure demonstrates the distribution of the signal intensity in the cross-section of the bent

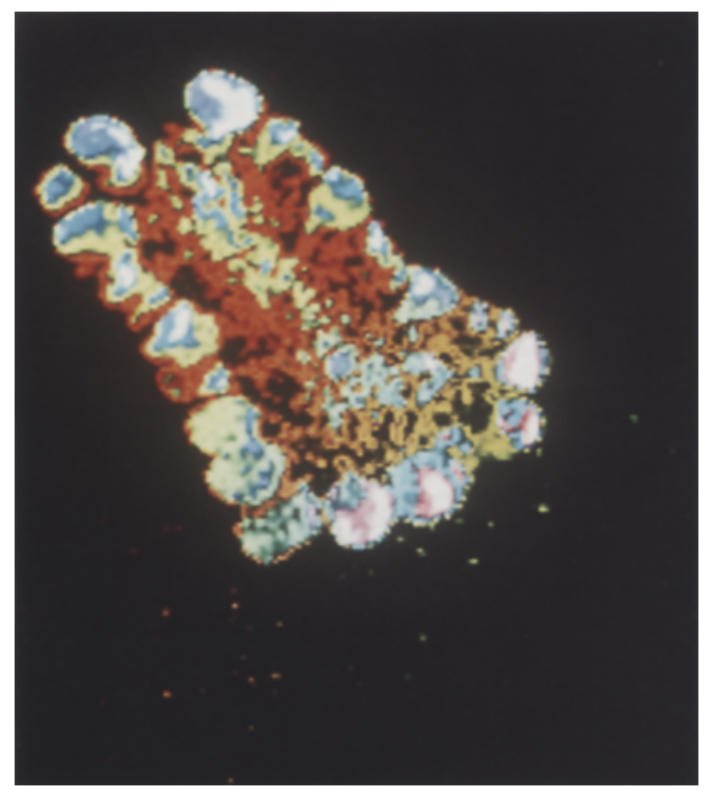

Fig. 2. A typical ${ }^{1} \mathrm{H}$ spin-echo NMR image of a intact leaf of Mesembryanthemum crystallinum in cross-sectional view at a spatial resolution of $20 \cdot 20 \cdot 100 \mu \mathrm{m}^{3}$. The spin-echo time was $18 \mathrm{~ms}$. The different signal intensities are shown as a falsecolour scale. White represents regions with mobile water, dark represents regions with strongly bound water or air gaps; intermediate colours correspond to regions of looser water-binding. In contrast to the bladder cells and the water-conducting vessels the water in the mesophyll cells seems to be bound. It is interesting to note that at least at this spin-echo time, black patches (low ${ }^{1} \mathrm{H}$-NMR signal intensity) are apparent within the relatively uniformly red-coloured mesophyll tissue. Magnification $=\times 15$ 


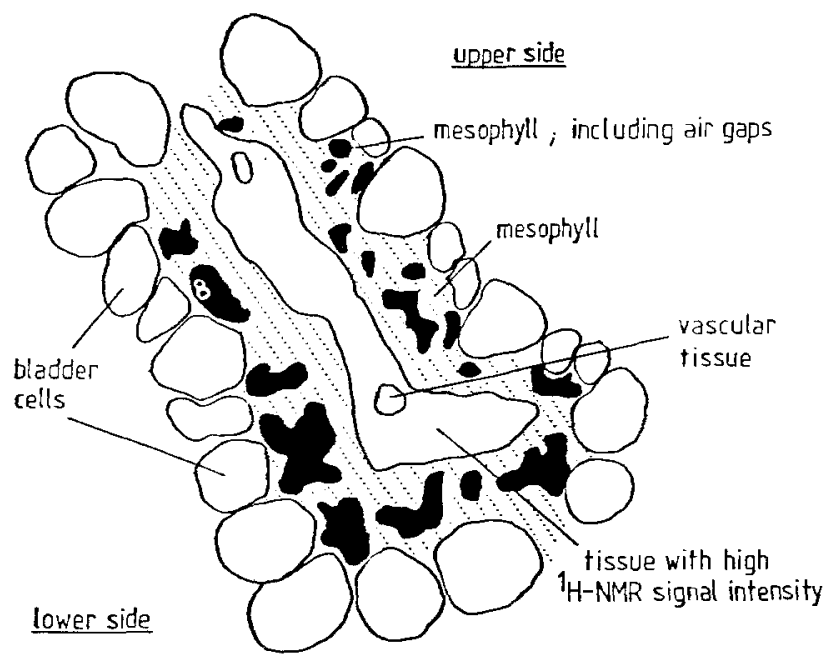

Fig. 3. A schematic interpretation of the image in Fig. 2. In the middle of the drawing the tissue with high ${ }^{1} \mathrm{H}-\mathrm{NMR}$ signal, including the vascular tissue, is shown (white region). Furthermore the two different mesophyll types (mesophyll with low signal intensity (black), mesophyll with higher signal intensity (stippled), and the bladder cells on the upper and lower surface of the leaf can be seen. Regions of mesophyll with low signal intensity are more extensive in the lower part of the leaf in comparison with the upper part. It was shown that these dark regions also contained air gaps

leaf as indicated in the schematic drawing of Fig. 3. The signals from the bladder cells and from the central water-conducting vessels are indistinguishable from free water. In addition, at higher magnifications (Fig. 4) water-conducting channels through the basal area of the bladder cells are clearly resolved. In contrast, the mesophyll tissue showed reduced signal intensity. The irregularly shaped dark-grey patches distributed in the mesophyll tissue indicated zones of very weak signal intensities. These zones were more frequently observed in the lower epidermis than in the upper one.

The long repetition time $\left(T_{R}\right)$ used for the NMR image reduced the influence of the spin-lattice relaxation time $\left(\mathrm{T}_{1}\right)$ on the signal intensity (see Theory). This implies that the differences in image intensity of the mesophyll tissues are due primarily to differences in the spin-spin relaxation time $\left(\mathrm{T}_{2}\right)$ and-or water content. Therefore, images were taken at spin-echo times $\left(\mathrm{T}_{\mathrm{E}}\right)$ of $18.24 \mathrm{~ms}$ and 4.4 ms (Fig. 5) in order to measure the regional distribution of water in the leaf (see Theory). At a $\mathrm{T}_{\mathrm{E}}$ of $4.4 \mathrm{~ms}$ the contrasts mainly correspond to an image of water concentration because the signals of regions with short $T_{2}$ values are accentuated. It is obvious that at the short $T_{E}$ of $4.4 \mathrm{~ms}$ the signal intensity was nearly uniform over the entire leaf with the exception of small (dark) areas which

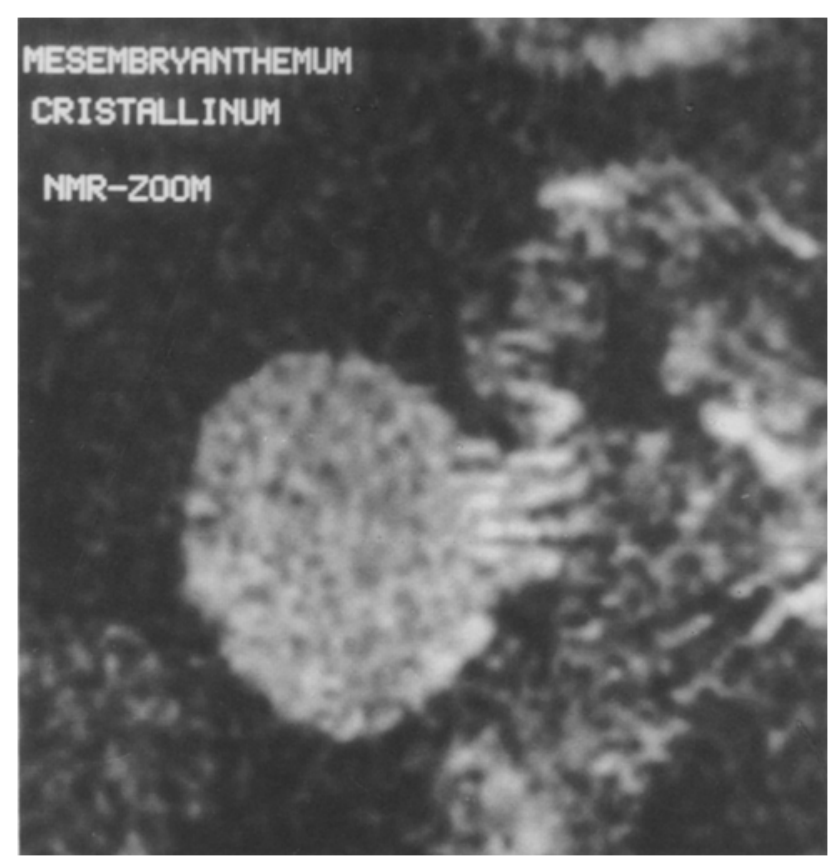

Fig. 4. An enlarged section of the NMR image ( $\mathrm{T}_{E}=18 \mathrm{~ms}$ ) of Fig. 2 shows a bladder cell which is symplasmatically connected to the mesophyll cells below it (spatial resolution: $20 \cdot 20$. $100 \mu^{3}$ ). The strands correspond to groups of plasmodesmata. Magnification $=\times 120$

were visible below the leaf surface in the gaps between the bladder cells. These areas were much smaller than the (dark) patches observed in the mesophyll tissue at $\mathrm{T}_{\mathrm{E}}=18 \mathrm{~ms}$ (see also Fig. 2). By analogy to the dark patches in Figs. 2 and 3 the frequency of these areas was higher at the lower epidermis than at the upper one. These areas did not apparently contain water and represented, therefore, air-filled spaces. The irregularly darkgrey patches observed at a $T_{E}$ of $18 \mathrm{~ms}$ must, therefore, reflect (apart from small air spaces) mesophyll cells in which water is more strongly bound than in the surrounding mesophyll cell tissue. Consistent with this view, vacuum-infiltration of the leaf with water $(17 \%$ increase in fresh weight per leaf) caused a large uniform increase in the image intensity at $T_{E}=18 \mathrm{~ms}$ associated with the disappearance of the contrast between the water-conducting vessels (and the bladder cells) and the mesophyll tissue (data not shown). The strong increase of mobile water in the leaf apparently masks the signals resulting from those mesophyll cells which may still contain bound water (data not shown).

The interpretation of the contrasting areas in the image of the mesophyll cell layer observed at $T_{E}=18 \mathrm{~ms}$ in terms of differences in bound water 


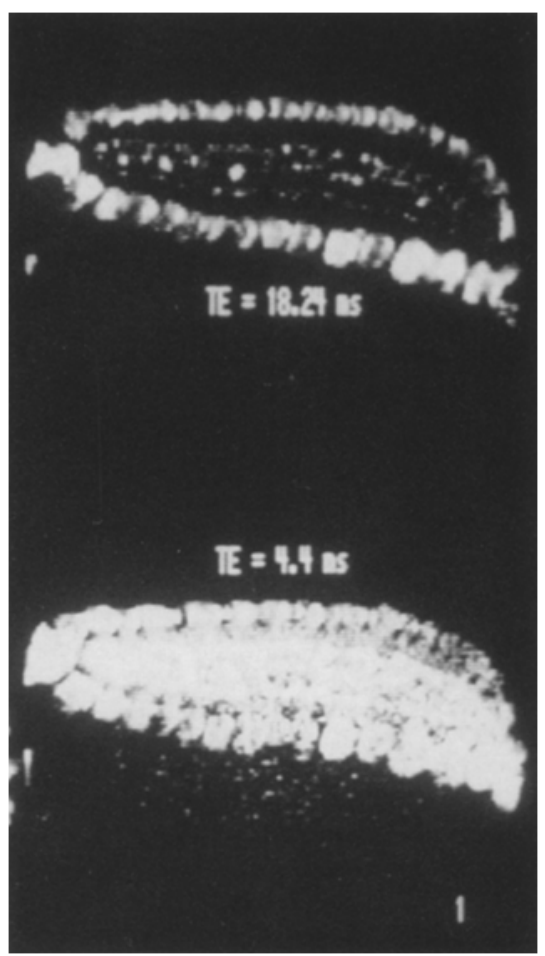

Fig. 5. Two ${ }^{1} \mathrm{H}$-NMR images of an intact leaf of $M$. crystallin$u m$ taken at different spin-echo times. At a $\mathrm{T}_{E}$ of $4.4 \mathrm{~ms}$ the image shows a nearly uniform signal intensity over the entire leaf with the exception of small dark areas. At this short $T_{E}$ the contrast mainly corresponds to an image of water concentration (see eqn. 2). The dark areas are much smaller than the dark patches observed in the mesophyll tissue at a $T_{E}$ of $18.24 \mathrm{~ms}$ (see also Fig. 2). They did not apparently contain water and represented, therefore, air-filled spaces (magnification $=\times 13$ ). Further explanations are given in the text

was verified by comparison with the image obtained by morphological investigations.

Horizontal and vertical cross-sections (made by hand or by microtome) showed that different types of mesophyll cells could be distinguished. As illustrated for example in the semi-thin cross-section in Fig. 6, large and uniform mesophyll cells were located close to the bladder cells (shrunken due to dehydration during the preparation), whereas in regions below the epidermis cells (which were located between the bladder cells) small and heterogeneous mesophyll cells were observed. The large mesophyll cells contained a low concentration of chloroplasts compared with the smaller ones (seen by cross-sections of fresh tissue, not shown).

In addition, in the regions of the smaller mesophyll cells many large intercellular spaces could be resolved, whereas the region containing the larger mesophyll cells exhibited only a few small air spaces. The number of these air spaces was larger in the lower epidermis than in the upper one.

The presence two types of mesophyll cells has not been reported in the literature up to now (Haberland 1918; Engmann 1934; Metcalfe and Chalk 1950; Kramer 1979), although at the beginning of this century Oberstein (1913) described similar structural differences between the mesophyll cells. However, further support for our and Oberstein's observations has been obtained from preliminary experiments (data not shown) in which mesophyll protoplasts were isolated from leaves of $M$. crystallinum. Purification of these protoplasts by sucrose density gradients revealed two protoplast fractions of different densities (average diameter 95.1 \pm 27.7 $\mu \mathrm{m}$ and $61.2 \pm 18.6 \mu \mathrm{m}$, respectively).

Because of the striking similarities between the NMR images and these results we can conclude that the differently contrasting areas of the NMR image at $T_{E}=18 \mathrm{~ms}$ of the mesophyll cell layer indicate cells in which water is more strongly bound than in others.

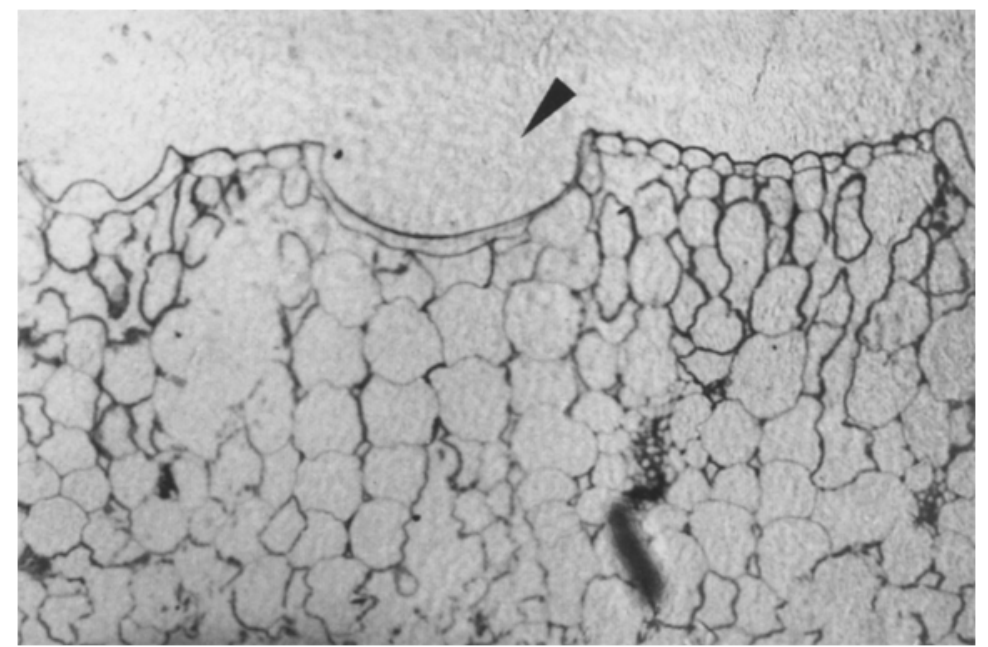

Fig. 6. Semi-thin cross-section ( $1.5 \mu \mathrm{m}$ thickness) of a leaf of $M$. crystallinum $L$. The epidermis on the margin of the leaf is interrupted by large bladder cells (which shrank during fixing; arrow) and a number of stomata with respiratory cavities below. The differentiation of the mesophyll into larger cells below the bladder cells and into smaller cells (accompanied by large intercellular spaces) below the epidermis can be recognized, even though the removal of water during preparation of the tissue was combined with some cell deformation. These deformations cannot be avoided because of the differences in osmolarity and cell turgor of the various leaf cells. Magnification $=\times 80$ 


\section{Discussion}

The ability to noninvasively measure relative water concentration and binding in living plant tissues represents a promising tool in the elucidation of the water relations of higher plants. Although this view is accepted by many authors (Bottomley et al. 1984; Aguayo et al. 1986; Eccles and Callaghan 1986; Johnson et al. 1987), so far the resolution of NMR images has been inferior to that obtainable by optical microscopy. However, the results reported here demonstrate that the use of high magnetic field strengths could bring the resolution of the NMR images to the level of a few cells and that additional variation of the spin-echo time $\left(T_{E}\right)$ allows the qualitative distinction between water concentration and water binding in different tissue cells.

The interpretation of the weak signal intensity in the mesophyll cell layer observed at $\mathrm{T}_{\mathrm{E}}=18 \mathrm{~ms}$ in terms of a high concentration of bound water molecules is in contradiction to the interpretation of Connelly et al. (1987). They explain low signal intensities in the cortex of a plant stem in terms of the diffusion of tissue water within the inhomogeneous magnetic-field gradients set up by air spaces in the tissue.

The attenuation of the intensity of spin-echo NMR signals under the influence of molecular diffusion within magnetic-field gradients is well known and has been applied to measure diffusion coefficients (Stejskal and Tanner 1965). A magnetic-field gradient strength of the order of 20 to 100 $\mathrm{mT} / \mathrm{m}$ has to be applied to give reasonable attenuation of the spin-echo signal intensity (Stejskal and Tanner 1965). However, air-containing regions surrounded by soft tissue normally give rise to magnetic-field gradients of the order of $1 \mathrm{mT} / \mathrm{m}$ (Haase, data not shown). Therefore the low signal intensity of the mesophyll cell layer cannot be explained in terms of molecular water diffusion in magnetic-field gradients.

The measurements at very short spin-echo times also exclude the possibility that the dark-grey patches observed at longer spin-echo times in the mesophyll cell layer represent intercellular spaces. The average size of the intercellular space observed in the optical images would also be inconsistent with this explanation because the mesophyll patches of low signal intensity at $T_{E}=18 \mathrm{~ms}$ cover a larger tissue area than the air spaces. The conclusion that water is apparently more bound in this mesophyll cell type than in other ones has important consequences for the interpretation of the water relations of the leaf of this plant. Rygol et al.
(1986, 1988) have recently studied the day-night rhythm in the turgor pressure of individual mesophyll cells and in single bladder cells of the upper and lower epidermes of salt-treated $M$. crystallinum plants by using the pressure probe (Zimmermann et al. 1969; Steudle and Zimmermann 1971; Hüsken et al. 1978; Zimmermann 1978, 1988). As a consequence of malate accumulation in the mesophyll cells during the night and the subsequent degradation of this compound, a maximum in turgor pressure is recorded in the mesophyll cells at the beginning of the light period $(06.00 \mathrm{~h})$. This maximum in turgor pressure is followed $6 \mathrm{~h}$ later by a pressure maximum in the bladder cells of the upper epidermis. In contrast, the turgor pressure in the bladder cells of the lower epidermis remains constant during the light-dark regime, except for some random fluctuations. Rygol et al. (1988) explained this finding by the assumption that water movement during (long-term) malate accumulation and degradation in salt-treated plants occurs primarily between the mesophyll cells and the bladder cells of the upper epidermis. On the other hand, the half-times of water exchange through the basal membranes of the bladder cells were identical for both groups of bladder cells (upper and lower) as revealed by injection of pressure pulses by means of the pressure probe. The NMR-image measurements reported here yield a consistent view of these apparently contradictory results. Figure 4 indicates that a nearly unrestricted water exchange between the individual bladder cells of the upper and lower epidermes (which function as water reservoirs; analysed by Steudle et al. 1975) and the first layer of mesophyll cells can occur through strands in the basal barrier of the bladder cells. According to Kramer (1979), the strands correspond to groups of plasmodesmata. The high signal intensity of the NMR image in this region for both bladder-cell types is a clear indication of the high water permeability of the barriers. This is also consistent with the unusually low values of the hydraulic conductivity and of the reflection coefficients measured for these membrane barriers with the pressure probe (Rygol et al. 1989). However, because of the presence of mesophyll cells which strongly bind water in deeper layers of the tissue, water transport due to osmotic changes during CAM may be hindered through the tissue. According to the uneven distribution of this water-immobilising mesophyll tissue this restriction should be more pronounced for the water exchange between the bladder cells of the lower epidermis than between the bladder cells of the upper epidermis and the underlying mesophyll cells. The lack of a pressure 
maximum in the bladder cells of the lower epidermis during the day-night rhythm may therefore result from the time-delayed water-transport properties of these special mesophyll cells. This explanation would also indicate that water is predominantly transported by the cell-to-cell pathway (as also proposed for barley roots by Steudle and Jeschke 1983).

This interpretation shows that NMR-image measurements can contribute to the understanding of the water relations of plant tissue even though this technique yields - at least at the present only relative information about water content and binding.

The authors are grateful to Dr. W.M. Arnold for critically reading the manuscript. This work was supported by funds from the Sonderforschungsbereich 176 (B4) and from the Forschergruppe Ökophysiologie, Universität Würzburg to U.Z. and by a Heisenberg Stipendium of DFG to A.H..

\section{References}

Aguayo, J., Blackband, S., Schoeniger, J., Mattingly, M., Hintermann, M. (1986) Nuclear magnetic resonance imaging of a single cell. Nature 322, 190-191

Bloch, F. (1946) Nuclear induction. Phys. Rev. 70, 460-474

Bottomley, P.A., Foster, T.H., Argersinger, R.E., Pfeifer, L.H. (1984) A review of normal tissue hydrogen NMR relaxation times and relaxation mechanisms from 1-100 MHz: Dependence on tissue type, NMR frequency, temperature, species, excision, and age. Med. Phys. 11, 425- 448

Bottomley, P.A., Rogers, H.H., Foster, T.H. (1986) NMR imaging shows water distribution and transport in plant root systems in situ. Proc. Natl. Acad. Sci. USA 83, 87-89

Connelly, A., Lohman, J.A.B., Loughman, B.C., Quiquampoix, H., Ratcliffe, R.G. (1987) High resolution imaging of plant tissues by NMR. J. Exp. Bot. 38, 1713-1723

Eccles, C.D., Callaghan, P.T. (1986) High-resolution imaging. The NMR microscope. J. Magn. Reson. 68, 393-398

Engmann, K.F. (1934) Studien über die Leistungsfähigkeit der sukkulenter Pflanzen. Beih. Bot. Cbl. 52a, 381-414

Fullerton, G.D., Potter, J.L., Dornbluth, N.C. (1982) NMR relaxation of protons in tissues and other macromolecular water solutions. Magn. Reson. Imag. 1, 209-228

Haberlandt, G. (1918) Physiologische Pflanzenanatomie. W. Engelmann Verlag, Leipzig

Hüsken, D., Steudle E., Zimmermann, U. (1978) Pressure probe technique for measuring water relations of cells in higher plants. Plant Physiol. 61, 158-163

Johnson, G.A., Brown, J., Kramer, P.J. (1987) Magnetic resonance microscopy of changes in water content in stems of transpiring plants. Proc. Natl. Acad. Sci. USA 84, 27522755

Kramer, D. (1979) Ultrastructural observations on developing leaf bladder cells of Mesembryanthemum crystallinum L.. Flora 168, 193-204

Lauterbur, P.C. (1973) Image formation by induced local interactions: Examples employing nuclear magnetic resonance. Nature 242, 190-191

Lüttge, U., Fischer, E., Steudle, E. (1978) Membrane potential and salt distribution in epidermal bladders and photosynthetic tissue of Mesembryanthemum crystallinum. Plant Cell Environ. 1, 121-129

Mansfield, P., Morris, P.G. (1982) NMR imaging in biomedicine. Academic Press, New York

Metcalfe, C.R., Chalk, L. (1950) Ficoidaceae. Anatomy of the dicotyledons, vol. I, pp. 706-711, Claredon Press, Oxford

Oberstein, O. (1913) Über den Bau der Blattspitzen der Mesembrianthema-Barbata. Beih. Bot. Cbl. 29, 298-302

Rygol, J., Büchner, K.-H., Winter, K., Zimmermann, U. (1986) Day night variations in turgor pressure in individual cells of Mesembryanthemum crystallinum L.. Oecologia 69, 171175

Rygol, J., Zimmermann, U., Balling, A. (1989) Water relations of individual leaf cells of Mesembryanthemum crystallinum at high and low external salinity. J Membr. Biol. 107, 203212

Schuhmacher, J.H., Clorius, J.H., Semmler, W., Hauser, H., Matys, E.R., Maier-Borst, W., Hull, W.E. (1987) NMR relaxation times $T_{1}$ and $T_{2}$ of water in plasma from patients with long carcinoma: Correlation of $\mathrm{T}_{2}$ with blood sedimentation rate. Magn. Reson. Med. 5, 537-547

Stejskal, E.O., Tanner, J.E. (1965) Spin diffusion measurements: Spin echoes in the presence of a time-dependent field gradient. J. Chem. Phys. 42, 288-292

Steudle, E., Jeschke, W.D. (1983) Water transport in barley roots. Planta 158, 237-248

Steudle, E., Lüttge, U., Zimmermann, U. (1975) Water relations of the epidermal bladder cells of the halophytic species $\mathrm{Me}$ sembryanthemum crystallinum: Direct measurements of hydrostatic pressure and hydraulic conductivity. Planta 126, 229-246

Steudle, E., Zimmermann, U. (1971) Hydraulische Leitfähigkeit von Valonia utricularis. Z. Naturforsch. 26b, 1302-1311

Winter, K. (1973) $\mathrm{CO}_{2}$-Fixierungsreaktionen bei der Salzpflanze Mesembryanthemum crystallinum unter variierten Außenbedingungen. Planta 114, 75-85

Winter, K. (1985) Crassulacean acid metabolism. In: Photosynthetic mechanisms and the environment, pp. 329-387, Barber, J., Baker, N.R., eds. Elsevier, Amsterdam

Zimmermann, U. (1978) Physics of turgor- and osmoregulation. Annu. Rev. Plant Physiol. 29, 121-148

Zimmermann, U. (1988) Determination of water relations of plant cells by means of the pressure probe technique. Methods Enzymol. (in press)

Zimmermann, U., Hüsken, D., Schulze, E.D. (1980) Direct turgor pressure measurements in different leaf cells of Tradescantia virginiana. Planta 149, 445-453

Zimmermann, U., Raede, H., Steudle, E. (1969) Kontinuierliche Druckmessung in Pflanzenzellen. Naturwissenschaften 56, 634-635

Received 9 November 1988; accepted 23 March 1989 